\title{
HEES: a Hexagon-based Energy Efficient Scheduling Protocol for Target Tracking in WSNs
}

\author{
Guowei Gu \\ Dept. of Computer Science and Technology \\ East China Normal University \\ Shanghai, China \\ e-mail: ggw_hsd@hotmail.com
}

\author{
Liyang Yu \\ Dept. of Computer Science and Technology \\ East China Normal University \\ Shanghai, China \\ e-mail: lyyu@cs.ecnu.edu.cn
}

\begin{abstract}
Target tracking is one of important applications of WSNs. In this paper, we propose a hexagon-based energy efficient scheduling protocol for target tracking (HEES) in WSNs. In this strategy, we use Hexagon idea to predict the next location and then form a dynamic cluster to track a target. And a new Sleep/Wake-up schedule method is used to improve the network lifetime. Simulation results show that the proposed method has better performance in energy efficiency which leads to a long-term monitoring and is suitable to target's random movement.
\end{abstract}

Keywords-WSN; Target Tracking; Scheduling; Dynamic clustering.

\section{INTRODUCTION}

WSNs consist of sensor nodes with limited processing, communication capabilities and scarce power. With the development in MEMS, the size and cost of sensor nodes are decreasing. WSNs have attracted much interest in the past decade. Wildlife tracking is one of the most important applications. In a wildlife tracking system, we can track one or more animals in the monitoring region and learn some animal behaviors from the animals' location and environment information. Energy saving is one of the biggest challenges in wildlife tracking. The devices attached to animals have less power than the other sensor devices which are deployed in the forest. In case of small animals, a device weighing more than $5 \%$ of the body weight is usually too heavy [1]. So the devices which be attached on the animals have lower power than other devices. To replace their batteries is much more difficult than the other devices. We should prolong their lifetime in order to tracking the animal for a long time as well as prolong the network lifetime.

In this paper, we propose the HEES which is a hexagonbased energy efficient scheduling protocol for target tracking in WSNs. We use the hexagon idea to select the sensor nodes which will stay in active mode and other nodes stay in sleep mode for saving energy in HEES.

The rest of the paper is organized as follows: In Section II, we discuss related works. In Section III, the proposed strategy is presented. In Section IV, we present and analyze the simulation results. In Section V, we conclude the paper.

\section{RELATED WORKS}

According to [2], three main approaches for target tracking in WSNs have existed: prediction-based method, tree-based method and cluster-based method. In tree-based method, nodes in a network may be organized in a hierarchical tree or be represented as a graph. In clusterbased method, nodes in a network should form clusters statically or dynamically.

In [2], a dynamic clustering method is proposed. This method use dynamic clustering method to track a target. It can reduce the energy consumption and prolong the network lifetime.

The prediction-based approach is built upon the treebased method or cluster-based method with special prediction methods [3]. This approach usually predicts next location of the target and then only select some nodes for tracking while other nodes stay in sleep mode for energy saving. There are many prediction-based methods such as DPT [4], PTPS [5]. The disadvantage of the approach is that it consumes much energy to finish the failure recovery when the target moves abruptly or changes the direction constantly.

\section{DESCRIPTION OF HEES}

\section{A. Assumptions of the HEES}

The following assumptions are made for the design of HEES:

- The sensors are all time synchronized.

- Every sensor node knows its own position.

- All the monitor sensor nodes have the same capabilities such as sensing, communication and data processing.

- All targets are assumed to move from the outside into the sensing range.

- Our location model is cell-based model.

- In our paper, we choose the hexagon for the shape of the hexagon is an idea shape. In [6], the detail analysis is given. In our paper, a hexagon is a cell.

\section{B. Network topology}

The network topology is showed in Fig. 1. A monitoring region is divided into many small hexagons. A small circle is a node, and a triangle is a sink. We attach hexagon center's position to the sensor node's position. It has two 
kinds of nodes, a border node and a non-border node. In Fig. 1, white nodes are non-border nodes, and the others are the border nodes. The border nodes should be active all the time in order to detect the targets that move into the sensing range. The distance between the nearest nodes is $R$. Every target sensor has a unique ID and the range of communication is $r$. It broadcasts its ID every $t$ second. After broadcasting its ID, it will stay in sleep.

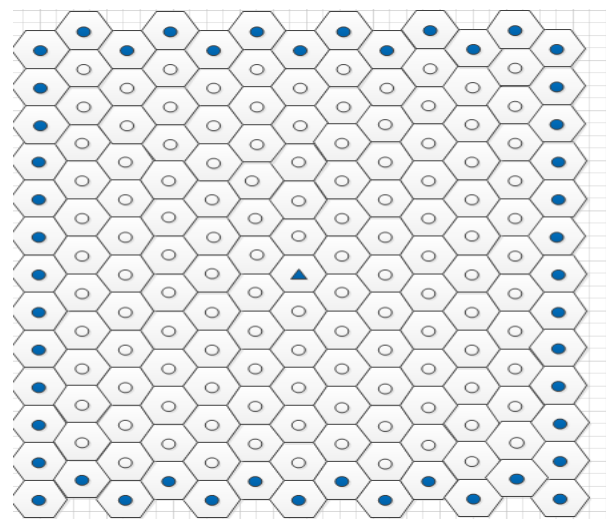

Figure 1. The network topology

\section{THE TRACKING METHOD OF HEES}

An obvious way to save energy is to switch on only a subset of the sensor nodes. In [7], four typical activation strategies are discussed. Here, we combine Duty-cycled activation (DA) and selective activation (SA) into our strategy. If a node is monitoring a target in the period of the duty-cycle, we called it a monitoring node (M-node). If not, we called it a sleeping node(S-node).

\begin{tabular}{c|c|c|c|c|} 
S-node & $\mathrm{T}_{\sigma}$ & $\mathrm{T}_{\rho}$ & $\mathrm{T}_{\eta}$ & $\mathrm{T}_{\mathrm{v}}$ \\
\hline M-node & $\mathrm{T}_{\mu}$ & $\mathrm{T}_{\rho}$ & $\mathrm{T}_{\eta}$ & $\mathrm{T}$ \\
\hline \multirow{3}{*}{ Figure 2. The DA strategy }
\end{tabular}

In Fig. 2, the DA strategy of our sensor nodes is given. $T_{\mu}$ : this stage is to detect a target and identify it.

$T_{\sigma .}$ this stage is to sleep.

$T_{\rho}$ : this stage is to report the target's position to a sink.

$T_{\eta}$ : this stage is to schedule the needed nodes to track the target.

$T_{v}$ : this stage is reserved and is to sleep now.

Our proposed tracking protocol (HEES) has three steps:

1) Detecting a target

The location model of HEES is a cell-based model. A target broadcasts a packet which contains its ID every $t$ seconds. $T_{\mu}=T_{\sigma}=t$. The sensing range of node is $\rho$. The maximum velocity of a target is $\varpi$. When a target moves into the sensing range of border nodes, HEES can detect a target by receiving a message from the target. HEES can only detect a target when $\varpi<\rho / t$. So we also assume that

$$
T=T_{\mu}+T_{\rho}+T_{\eta}+T_{\nu}=\rho / \varpi
$$

Equation (1) means that an M-node should spend $T_{\mu}$ seconds to detect a target at least once a time of $T$ and it takes a target at least $T$ seconds to pass through a cell. $T$ is called a dutycycle period.

\section{2) Reporting a target's position.}

Once a node detects a target, it will send its position to the sink in the $T_{\rho}$ stage of DA. Many location-based protocol of WSN can be applied. This is not our focus.

3) Scheduling nodes to track the targets and infer the position of the target.

When a border node detects a target, it needs to activate some nodes to keep tracking. How to select the node can affect the total energy consuming of the network. If the target is a human, prediction-based target tracking methods will work efficiently because the moving of people has some obvious, inherited regularity. Prediction-based methods are not suitable for the target moving abruptly or changing the direction constantly. If prediction is wrong, it will take some time to find the lost target and will consume much energy.

The sensor nodes can be scheduled efficiently in our proposed protocol no matter whether the target has inherited regularity or not. It combines dynamic clustering method and prediction method to scheduling some nodes to track some targets.

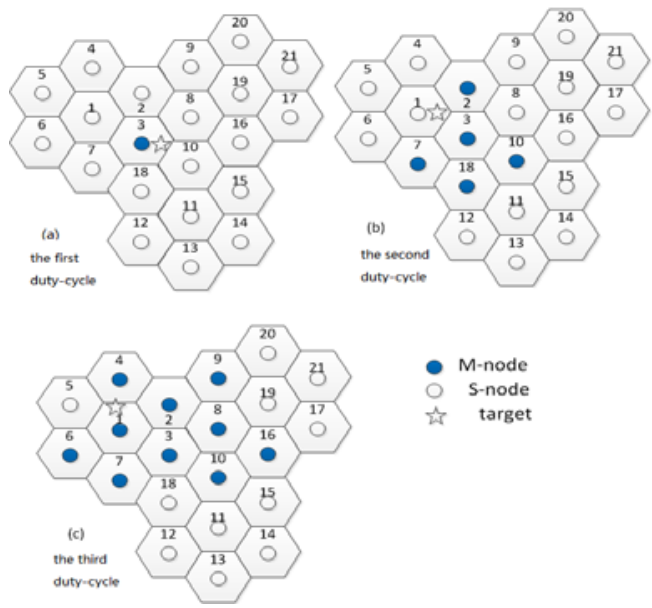

Figure 3. Scheduling strategy

\section{B. Selective activation(SA)}

\section{1) Scheduling Message}

Our SA strategy is our scheduling method. We assume that direction angle is based on east, i.e. the direction angle of east is zero.

\section{2) SA strategy}

Firstly, when a target moves into the monitoring region, it will be detected by a border node. We assume that this time is in the first duty-cycle period. When a node detects a target, it will broadcast a one-hop message which contains its detected target ID and detected receive signal strength. The receive signal strength is used to determine who is closest to the target. After this period, an M-node is found and become a temporary cluster head.

Secondly, the M-node reports the target's position to a sink and schedules the nodes who should be M-nodes in the 
next DA period. If the cluster head is a border node, it will broadcast a scheduling message to its neighbors, and all his neighbors will become M-node in the next DA period. The border node's SA strategy is simple. All its neighbors are scheduled to be M-nodes. These M-nodes stay in active mode for a DA period, and then become S-nodes except border nodes. Border nodes should be M-nodes all the time.

If the cluster head is a non-border node, it will schedule some nodes and make some nodes become his cluster members by using prediction method and clustering method.

The prediction method is that using the last detected position and the cluster head's position to predict the next position after the DA period. And then, a cluster head will broadcast a two-hop scheduling message which contains a pattern field, cluster head's position field. The direction angle from the cluster head to the predicted position is set to pattern. Here, we define that the angle between the directed line from the cluster header to a node and the directed line which pattern can depict is called $\alpha$.

If one neighbor node's $\alpha$ is 120 degrees, this neighbor node will become a M-node in the third DA period for one DA period. If $\alpha$ is zero degree, it will become an M-node in the next period and turn to an S-node in the third DA period. Other neighbor nodes will become M-node in the second DA period and keep this mode for two DA periods.

If a node is two-hop away from the cluster head and its $\alpha$ is equal to 90 or 150 , it will turn to M-node in the third DA period and stay in active mode for a DA period. Other twohop nodes only drop the message.

If an M-node detects the target in the next DA period, it will send an ack message to a node and become a new temporary cluster head. When the old cluster head receive the ack message, it will send a cancel message which makes the members and itself to be S-nodes. The new cluster head will make a prediction and form a new cluster.

Every cluster head and cluster members will be S-nodes after two DA periods. So a cluster member needn't send an ack message when it detects the target in the third DA period.

In Fig. 3, the scheduling strategy is shown.

Node 3 detected a target at time $t_{1}$ and last position is node 2 . Node 3 predicts that target maybe at node 18 . So we predict the target direction angle is 270, and set pattern 270 . Node 3 broadcasts a scheduling message to its neighbors. And node 1 and node 3 will forward this message to their neighbors.

In the next duty-cycle period, node $2,7,18,10$ become M-nodes. If no target is detected by any M-node, node 1, 4, 6 , $9,18,16$ will become $\mathrm{M}$-nodes and node 18 will become Snode in the third duty-cycle period because of its $\alpha$ is zero. At time $t_{2}$, a target can be detected by one M-node. If the target is detected by node 1 , we can calculate that the nodes in the target's moving path are node 3 at $t_{1}$, node 1 at $t_{2}-T$ and node 1 at $t_{2}$.

\section{Analysis}

On the assumption that speed isn't constant and direction changes constantly, we compare our method with prediction method and dynamic clustering method in our network scenario by using probability theory.
The maximum velocity of a target is $\varpi$. So we can ensure that recovery energy consume of prediction-based method can be analyzed. We assume that a target moves randomly. The probability of a target moving to a next nearest cell, $\mathrm{P}(\mathrm{m})$, is $1 / 7$ (maybe it doesn't move). Let $N_{n}$ be the average number of nodes which need to monitor a target successfully. Let $C$ be the number of cells which the target passed through.

Condition probability is denoted mathematically as (2),

$P(A \mid B)=$ (the number of times $A$ and $B$ can occur) / (the number of times $B$ can occur)

Bayesian rule is given in (3). Event $A$ is dependent on a number of mutually exclusive events $B_{1}, B_{2}, \cdots \cdots B_{n}$.

$$
P(A)=\sum_{i=1}^{n} P\left(A \mid B_{i}\right) \times P\left(B_{i}\right)
$$

So we can use (4) and (5) to calculate the $N_{n}, C$. The $w_{i}$ is the number of nodes which need to be awake to monitor a target in event $B_{i}$. $B_{i}$ represents that some node detects the target. $C_{i}$ is the number of cells which the target passes through in event $B_{i}$. Equation (6) shows that the average number of nodes which the target passing a cell needs to activate.

$$
\begin{aligned}
& N_{n}=w_{t} \sum_{i=1}^{n} P\left(A \mid B_{i}\right) \times P\left(B_{i}\right) \\
& C=C_{t} \sum_{i=1}^{m} P\left(A \mid B_{t}\right) \times P\left(B_{t}\right)
\end{aligned}
$$

$$
N_{c}=N_{n} / C
$$

We use (4), (5), (6) to get the $N_{c}$ of the prediction-base method, dynamic clustering method and our strategy. The result is showed in table I.

TABLE I. THE RESULT OF ANALYSIS

\begin{tabular}{|c|c|c|c|}
\hline Strategy & $\boldsymbol{N}_{\boldsymbol{n}}$ & $\boldsymbol{C}$ & $\boldsymbol{N}_{\boldsymbol{c}}$ \\
\hline Prediction-based & 7.85 & 1 & 7.85 \\
\hline Dynamic clustering & 7 & 1 & 7 \\
\hline HEES & 7.85 & 1.28 & 6.13 \\
\hline
\end{tabular}

TABLE II. SIMULATION SETTING

\begin{tabular}{|c|c|}
\hline Number sensors & 336 \\
\hline Monitored region & $400 \times 500$ meter $^{2}$ \\
\hline Sensor coverage range & $15 \mathrm{~m}$ \\
\hline Simulation duration & $660 \mathrm{~s}$ \\
\hline Sensor communication range & $30 \mathrm{~m}$ \\
\hline MAC layer & $802.11 \mathrm{~b}$ \\
\hline Network topology & Fig. 1 \\
\hline
\end{tabular}

By analyzing $N_{c}$, the average number of nodes which needs to be awake to track a target when the target passes a cell, we get that our strategy has a better performance than others. 


\section{PERFORMANCE EVALUATION}

In this section, we evaluate the performance of HEES with the Naïve Activation method (NA) [7] and the dynamic clustering method [2]. NA is a useful baseline for comparison because it provides the best possible quality of tracking. The tool that is used for simulation is NS-2. Firstly, we describe the settings for our simulation. Secondly, we introduce the metrics considered to comparing the performance. Finally, we evaluate the performance by comparing three methods. Our simulation settings are showed in Table II. The mobility model is implemented through configuration files manually. The sensor model is the model of WINS [8].

In order to evaluate the performance, the follow metrics will be used:

\section{- $\quad$ Target energy consumption}

- $\quad$ Total energy consumption

Fig. 4 shows the total energy consumption of three strategies for different velocities of a target when the target broadcasting interval is one second. We can learn that HEES can consume less energy than the others.

Fig. 5 shows that the target's energy consumption is very little. And it can work for a long time in the current mode.

We can also learn that as velocity increases, energy consumption of HEES become closer to the dynamic method. This is because the increase of target's velocity leads to the DA period (i.e. T) becoming shorter and the difference between $T$ and $T_{\mu}$ become small. It will also lead to the decline of the ratio of the sleep time $T_{v}$ to to the DA period $T$.

\section{CONCLUSIONS}

This study has presented a target tracking method which is energy efficient and suitable to target's random movement. We performed theoretical analysis to compare HEES with the prediction method and dynamic clustering method. And we also performed simulations to compare the performance

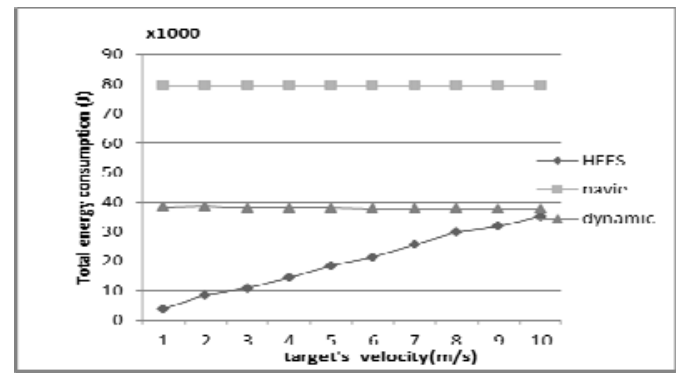

Figure 4. Total energy consumption in different maximum velocities of HEES with navie method and dynamic method. Simulation results demonstrated that HEES has low energy consumption, high tracking precision even though the velocity and moving direction of a target varies rapidly. Our future work will focus on finding a method to make this protocol be suitable in the random deployment region.

\section{REFERENCES}

[1] Vladimir Dyo1, Stephen A. Ellwood2, David W. Macdonald, "Poster Abstract: Wildlife and Environmental Monitoring using RFID and WSN Technology" in Proceeding SenSys '09 Proceedings of the 7th ACM Conference on Embedded Networked Sensor Systems, Nov. 2009, pp. 371-372.

[2] Guang-yao Jin, Xiao-yi Lu, and Myong-Soon Park, "Dynamic Clustering for Object Tracking in Wireless Sensor Networks", Ubiquitous Computing Systems, Seoul, Korea, Volume 4239, 2006, pp. 200-209.

[3] Sania Bhatti, Jie Xu, "Survey of Target Tracking Protocols using Wireless Sensor Networks”, Wireless and Mobile Communications, 2009. ICWMC '09. Fifth International Conference on, Aug. 2009, pp. 110-115

[4] H.Yang, B.Sikdar, "A Protocol for Tracking Mobile Targets using Sensor Networks”, Sensor Network Protocols and Applications, 2003. Proceedings of the First IEEE. 2003 IEEE International Workshop on, May, 2003, pp. 71-81.

[5] Samer Samarah, Muhannad Al-Hajri, and Azzedine Boukerche, “An Energy Efficient Prediction-based Technique for Tracking Moving Objects in WSNs”, Communications (ICC), 2011 IEEE International Conference on, June. 2011, pp. 1-5.

[6] Hady S.AbdelSalam, Stephan Olariu "HexNet: Hexagon-based Localization Technique for Wireless Sensor Networks”, Pervasive Computing and Communications, 2009. PerCom 2009. IEEE International Conference on, March. 2009, pp. 1-6.

[7] Sundeep Pattem, Sameera Poduri, Bhaskar Krishnamachari ,"EnergyQuality Tradeoffs for Target Tracking in Wireless Sensor Networks”, Information Processing in Sensor Networks, Volume 2634, 2003, pp. 32-46.

[8] Oscar Garcia, Alejandro Quintero, Samuel Pierre, "a global profilebased algorithm for energy minimization in object tracking sensor networks”, Computer Communications, Volumn 33, April. 2011, pp. 736-744.

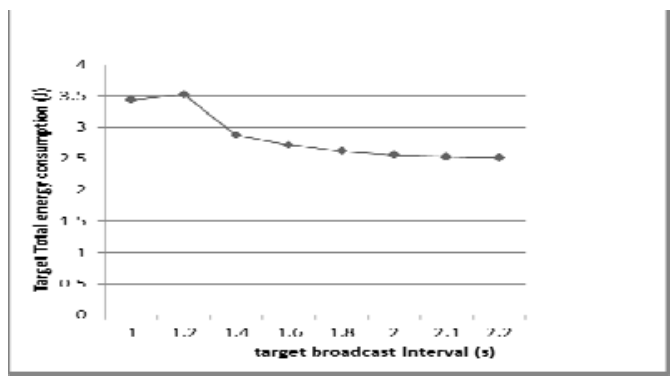

Figure 5. Target's energy consumption of HEES 